\title{
Acute accidental inhalation of Chlorine gas: A mini review
}

\author{
Franco Lai, ${ }^{1}$ Alessio Baldini, ${ }^{1}$ Luca Becheroni, ${ }^{1}$ Iacopo Cappellini, ${ }^{2}$ Barbara Balzarini, ${ }^{3}$ \\ Francesco De Antoniis, ${ }^{1}$ Alessandra Ieri, ${ }^{3}$ Francesco Gambassi, ${ }^{3}$ Chiara Pagnini, ${ }^{4}$ \\ Lorenzo Pelagatti, ${ }^{1,5}$ Mario Rugna, ${ }^{6}$ Simone Magazzini ${ }^{7}$
}

${ }^{1}$ Emergency Department, Ospedale S. Stefano, Azienda USL Toscana Centro, Prato; ${ }^{2}$ Intensive Care Unit, Ospedale S. Stefano, Azienda USL Toscana Centro, Prato; ${ }^{3}$ Toxicology Unit \& Poison Control Centre, Azienda Ospedaliero Universitaria Careggi, Florence; ${ }^{4}$ Intensive Care Unit, University of Siena, Siena; ${ }^{5}$ Emergency Department, Azienda Ospedaliero Universitaria Careggi, Florence; ${ }^{6}$ SOSD for Education Emergency Medical System 118, Azienda USL Toscana Centro, Florence; ${ }^{7}$ Emergency Departmental Area, Azienda USL Toscana Centro, Florence, Italy

\begin{abstract}
The Authors report an accidental gas exposure of Chlorine gas in a worker. This accident is very uncommon and can lead to important life-threatening conditions, such as Reactive Airway Disfunction Syndrome (RADS) and Acute Respiratory Distress Syndrome (ARDS) with important pulmonary disfunctions and even death. This syndrome results are reversible when a quick and appropriate intensive treatment is performed.
\end{abstract}

\begin{abstract}
Highlights
- Chlorine gas was first used as a chemical weapon on a largescale during World War I, and the War in Iraq. Other sources of chlorine gas exposure are workplace or industrial accidents: hypochlorite is available in many detergents and chlorinebased bleach, and when mixed with household acids, it releases chlorine gas.

- Most common symptoms include irritant effect on mucous membranes and may produce wheezing and other respiratory symptoms as cough, upper respiratory irritation, dyspnoea, stridor and bronchospasm.

- Pneumonitis, which can progress to Acute Respiratory Distress Syndrome (ARDS) may develop in severe exposure.

- Treat patients following the ABCDE approach.
\end{abstract}

Correspondence: Franco Lai, Emergency Department, Ospedale S. Stefano, Azienda USL Toscana Centro, Via Suor Niccolina $n^{\circ} 22$, Prato, Italy. E-mail: dott.francolai@gmail.com.

Key words: Chlorine gas exposure; Chlorine gas intoxication; RADS; ARDS.

Conflict of interest: The authors declare no conflict of interest.

Availability of data and materials: All data generated or analyzed during this study are included in this published article.

Ethics approval and consent to participate: All patients participating in this study signed a written informed consent form for participating in this study.

Informed consent: Written informed consent was obtained from a legally authorized representative(s) for anonymized patient information to be published in this article.

Received for publication: 27 June 2021.

Revision received: 15 November 2021.

Accepted for publication: 22 November 2021.

This work is licensed under a Creative Commons Attribution 4.0 License (by-nc 4.0).

${ }^{\circ}$ Copyright: the Author(s), 2021

Licensee PAGEPress, Italy

Emergency Care Journal 2021; 17:9941

doi:10.4081/ecj.2021.9941

\section{Case Report}

A 64 years-old male was admitted to the Emergency Department (ED) after accidental occupational inhalation of Chlorine 60 gas 56\% chlorine-based salt used in wool and fabric processing. The patient had been exposed for about 15 minutes to chlorine vapours and the symptoms, mainly of the upper respiratory tract (dysphonia and stridor) would have arisen after a few minutes of contact. The territorial emergency system was immediately alerted and after about 30 minutes the patient was being evaluated in the ED. Upon admission to the Emergency Room (ER), the patient was alert but presented dyspnoea, dysphonia, laryngeal stridor, profuse sialorrhea and a low peripheral oxygen saturation $\left(\mathrm{SpO}_{2}\right)$. His blood pressure was $105 / 60 \mathrm{mmHg}$, his heart rate 80 bpm, body temperature was $96.4^{\circ} \mathrm{F}$, with a Glasgow Coma Scale (GCS) of 15 and a $\mathrm{SpO}_{2} 93 \%$. Chest auscultation revealed diffuse decreased breath sounds and wheezing. An arterial blood gas analysis showed a pH of 7.409, $\mathrm{PaO}_{2} 52 \mathrm{mmHg}, \mathrm{PaCO}_{2} 68 \mathrm{mmHg}$, $\mathrm{HCO}_{3}-22 \mathrm{Mmol} / \mathrm{L}$, and $0.5 \mathrm{mmol} / \mathrm{L}$ lactate room air. Complete blood count showed haematocrit 42.7\%, MCV 92.6 fL, MCH 99.9 pg, MCHC 32.3 g/dL, RDW 13.2\%, MPV 10 fL, leukocyte count $8.3 \times 10^{9} / \mathrm{L}$ (neutrophils $82.4 \%$, lymphocytes $9.3 \%$, monocytes $7.3 \%$, basophils $0.6 \%$, eosinophils $0.4 \%$ ) and $210 \times 10^{9} / \mathrm{L}$ platelets. Glucose was $102.0 \mathrm{mg} / \mathrm{dL}$, total bilirubin $19 \mu / \mathrm{L}$, LDH was increased (290 U/L), as well as Fibrinogen (659 mg/dL) and CRP (1.06 mg/dL), PTT was 29 seconds, Sodium was $140 \mathrm{mEq} / \mathrm{L}$. Renal function, urea and troponin were normal (CKD-EPI was 70, troponin $18.2 \mathrm{mg} / \mathrm{dL}$, urea $29 \mathrm{mg} / \mathrm{dL}$ ). Negativity to Sars-Cov $\mathrm{C}_{2}$ was 
established through a nose pharyngeal swab. No pathological changes were observed on the ECG.

A prompt sedation and analgesia were induced with PROPOFOL $1,5 \mathrm{mg} / \mathrm{kg}$ and FENTANYL100 mcg intravenously (iv). Then, a paralytic agent, ROCURONIUM $50 \mathrm{mg}$ iv, was administered to facilitate advanced airway management. Endotracheal intubation and aspiration were performed, and invasive mechanical ventilation was then started. Next step was the insertion of central venous catheter and arterial cannulation to achieve advance monitoring of hemodynamic and respiratory parameters.

After initial treatment in the ER, the patient conditions were stable, mainly in gas exchange $\left(\mathrm{pH} 7.46, \mathrm{PaO}_{2} 98 \mathrm{mmHg}, \mathrm{PaCO}_{2}\right.$ $\left.\mathrm{mmHg}, \mathrm{HCO}_{3}-26 \mathrm{Mmol} / \mathrm{L}\right)$. Then, the patient was transferred to Intensive Care Unit (ICU) for the specific treatments. After two days of ICU, the patient showed a gradual improvement in both respiratory and haemodynamic parameters and was therefore extubated and finally transferred to medical setting.

As suggested by the Florence Toxicology Unit and Poison Control Centre specialist, we administered Methylprednisolone $125 \mathrm{mg}$ iv, Atropine $1 \mathrm{mg}$ iv, Salbutamol endotracheal and Omeprazole $40 \mathrm{mg}$ iv.

This therapeutic management is definitely in line with protocols proposed in literature. In case of intoxication with chlorine gas, the use of bronchodilators associated with corticosteroids is commonly accepted. In our case, moreover, atropine found its rational as a therapy for the profuse sialorrhea.

The patient underwent a chest and abdomen CT scan with iodinated contrast medium, whit finding of limited parenchymal thickening in left lung lower lobe, no signs of pneumothorax, pneumomediastinum or pleural-pericardial effusion. Spleen, pancreas and kidneys were regular. Mediastinal and retroperitoneal lymphadenopathy were absent. An ecstatic appearance of both common iliac arteries was observed.

\section{Discussion}

The victim was manipulating a bucket of Chlorine (Zawa $\mathrm{GmbH}$ ), a biocide containing $3 \mathrm{~kg}$ of pure sodium 1,1-dichlor-isocyanurat dihydrate (chlorine $56 \%$ ), in a soluble granular form, useful to degrease and wash the shearer wool first to be colored and spun. The product's composition was not registered in European Chemical Agency (ECHA) database, neither in the Istituto Superiore di Sanità (ISS) database (Archivio Prodotti Pericolosi), disregarding UE REACH and CLP regulations. Furthermore, its MSDS (Material Safety Data Sheet) was not available on the web, and the label only in German language, with pictograms referring generic health hazard and hazardous to environment.

Sodium 1,1-dichlor-isocyanurate dihydrate is an agent related to hypochlorite by the toxicological point of view. Most household bleach solutions contain 3\% to 5\% hypochlorite, while swimming pool disinfectants and industrial strength cleaners may contain up to $20 \%$ hypochlorite. The manual addition of an acid to a hypochlorite solution may release chlorine gas, while the ammonia may react with hypochlorite solutions to release chloramine, a gas with properties like chlorine. This scenario most often develops when several different household cleaning products are mixed.

In case of contact with chlorine, it should be added that ingestion of dilute (3\% to $5 \%$ ) hypochlorite liquid solutions will cause immediate burning in the mouth and throat, but no further injury would be expected, while more concentrated solutions may cause significant oesophageal and gastric burns, and patients may mani- fest dysphagia, drooling, and severe throat, chest, and abdominal pain. Hematemesis and gastrointestinal perforations can occur.

\section{Brief toxo-dynamic consideration}

Chlorine gas is an intermediate water-soluble irritant gas. After contact with airways, it dissolves with $\mathrm{H} 2 \mathrm{O}$ present on mucous membranes of airways responsible of irritant and corrosive local toxic effects and respiratory symptoms (Irritant Gas Syndrome).

Chlorine is an irritant gas whose toxicity depends on concentration, duration of exposure, sex of the exposed victims, their susceptibility due to underlying lung disease and smoking. ${ }^{1,2}$

Acute effects range from irritation of the conjunctivae and upper respiratory airways to acute lung injury, which can lead to pulmonary obstruction, reactive airway dysfunction syndrome, acute respiratory distress syndrome and, rarely, death..$^{3-7}$

The toxicity of chlorine is due to its oxidizing potential, and higher solubility in water. Once inhaled, chlorine is hydrolysed to hydrochloric acid and hypochlorous acid $(\mathrm{HOCl})$ and hypochlorite. These species of strong oxidants lead to bronchoalveolar inflammation and bronchoconstriction. At low concentrations (40 ppm) chlorine, distal areas of lungs are reached, leading to pulmonary edema, toxic pneumonitis and death, if the exposure is too long (>30 min at $430 \mathrm{ppm}$ ) or too intense (>1000 ppm). $)^{6,8,9}$

Long-term damage may occur with migration and activation of inflammatory cells, within the airway epithelium. ${ }^{5}$ The frequency of chlorine accidents and post-chlorine exposure management is of major public health concern. ${ }^{10-16}$

In 2014, the American Association of Poison Control Centers reported that about 6,000 exposures to chlorine gas in the US in 2013, compared with 13,600 exposures to carbon monoxide, which was the most common poison gas exposure; the year before they reported about 5,500 cases of chlorine gas poisoning compared with around 14,300 cases of carbon monoxide poisoning. ${ }^{17}$

Chlorine gas was first used as a chemical weapon on a largescale during World War I in the Second Battle of Ypres and is considered a choking agent. Protocol by the League of Nations prohibited the use of chemical and biological weapons. ${ }^{3}$ However, chlorine gas was again used by insurgents during the Iraq War and on a larger scale during the Syrian Civil War. Other sources of chlorine gas exposure are workplace or industrial accidents. The largest accident so far happened on 6 January 2005, in Graniteville, South Carolina, USA. There, two trains collided, one of them transporting chlorine gas, sodium hydroxide and cresol. Approximately 60 tons of chlorine gas were released. A total of 529 people sought medical care in local EDs, further 311 patients were treated by local physicians and another approximately 220 patients developed mild symptoms but did not seek treatment. ${ }^{6}$ Many victims with initially only mild complains had symptoms like cough and shortness of breath from a prolonged period of time, up to 8-10 months post exposure. ${ }^{5,6}$

Hypochlorite is available in many detergents and chlorinebased bleach, and when mixed with household acids, it releases chlorine gas. Therefore, household accidents with chlorine gas exposure are common. Furthermore swimming-pool tablets are often based on chlorine. The American Association of Poison Control Centers reports 4.305 chlorine gas exposures and another 2.284 from mixing hypochlorite with acids in 2017 alone with one reported fatality. ${ }^{7}$

Florence PCC (2018-2021) registered 272 exposures, out of these $92 \%$ originated mixing bleach with acids for household cleaning improperly, while only $8 \%$ by professional use. According to Poison Severity Score (PSS), 7\% of exposed had no problems, $46 \%$ showed mild, $15 \%$ moderate and $1 \%$ severe symp- 
toms with no deaths. Sixty-two percept $(62 \%)$ of the victims showed ocular and upper way irritation, 15\% dyspnoea, 5\% asth$\mathrm{ma} /$ bronchoconstriction. PCC suggested in $37 \%$ observation at home, in $26 \%$ a medical valuation, in $24 \%$ a short observation in ED, in $11 \%$ an hospital admission. Symptomatic/supportive therapy was disposed in $75 \%$ of cases (Tables 1,2 , and 3 ).

\section{Symptoms}

Most common symptoms include irritant effect on mucous membranes (e.g., eyes, nose, and throat) ${ }^{1}$ and may produce wheezing and other respiratory symptoms as cough, upper respiratory irritation, and dyspnoea, particularly in pre-existing diseases such as asthma or COPD. In serious exposures with household products, ${ }^{2}$ upper airway oedema may cause obstruction, and chemical pneumonitis ${ }^{3}$ may also occur as decreases in FVC, FEV1, and peak expiratory, persistent decreases in pulmonary function airway. Stridor and bronchospasm may develop after chlorine or chloramine inhalation or ingestion of sterilizing tablets. ${ }^{4}$ Exacerbation of asthma occurred in 1 case exposed to chlorine gas generated from the mixing of sodium hypochlorite and phosphoric acid Pneumonitis, which can progress to Acute Respiratory Distress Syndrome (ARDS). ${ }^{5}$ Pulmonary oedema, which can progress to acute hypoxemic respiratory failure, can occur from chlorine gas inhalation. ${ }^{6,7}$ Criteria for acute hypoxemic respiratory failure has included severe hypoxemia despite oxygen administration, chest x-ray showing diffuse pulmonary infiltrates, decreased lung compliance with intrapulmonary shunt, and normal cardiac function despite low (less than $18 \mathrm{mmHg}$ ) pulmonary artery occlusive pressure. Pneumomediastinum developed in 2 patients who inhaled chlorine gas after mixing sodium hypochlorite with acid. ${ }^{8}$

Supraglottic oedema induces shortness of breath, throat tightness and inability to speak. Rapid sequence intubation is mandatory in secondary to upper airway oedema. Severe respiratory distress requires intubation. For ingestions of hypochlorite solutions greater than $10 \%$ or symptoms of severe corrosive injuries (i.e., dysphagia, drooling, pain), flexible endoscopy should be performed to evaluate the extent of oesophageal or gastric injury. Chest and abdominal X-rays may be useful to look for mediastinal or intraabdominal free air secondary to perforations in the gastrointestinal tract, which require surgical intervention.

Patients with minimal occasional exposures who remain asymptomatic or develop mild symptoms with spontaneous resolution may remain home.

All patients with persistent symptoms or intentional exposures should be sent to a health care facility for observation for the longer of 4 to 6 hours or until symptoms resolve. Criteria for dis-

Table 1. Distribution of calls for chlorine gas exposures by year, proveniences and circumstances arrived to Toxicology Unit \& Poison Control Centre, Azienda Ospedaliero Universitaria Careggi, Florence, Italy.

\begin{tabular}{|c|c|c|c|c|c|c|c|}
\hline $\begin{array}{l}\text { Poison } \\
\text { Year }\end{array}$ & $\begin{array}{l}\text { ontrol Centr } \\
N \text { of cases }\end{array}$ & $\begin{array}{l}\text { orence: Chlorine Gas Exp } \\
\text { Provenience of the calls }\end{array}$ & $\begin{array}{l}\text { Ne } 2018-20 \\
N \text { of calls }\end{array}$ & $\%$ & Circumstances of exposure & $\mathrm{N}$ of cases & $\%$ \\
\hline 2018 & 77 & Private citizens & 95 & 34.9 & Houseold & 252 & 92.6 \\
\hline 2019 & 69 & Family Doctor & 4 & 1.5 & Other & 20 & 7.4 \\
\hline 2020 & 88 & Emergency Rescue & 44 & 16.2 & & & \\
\hline 2021 & 36 & Emergency Department & 128 & 47.1 &  & & \\
\hline TOTAL & 272 & & 272 & 100 & & 272 & 100 \\
\hline
\end{tabular}

Table 2. Distribution of exposure by poison severity score ${ }^{18}$ and symptoms in patient admitted to Toxicology Unit $\&$ Poison Control Centre, Azienda Ospedaliero Universitaria Careggi, Florence, Italy between $1^{\text {st }}$ January 2018 and $31^{\text {st }}$ October 2021.

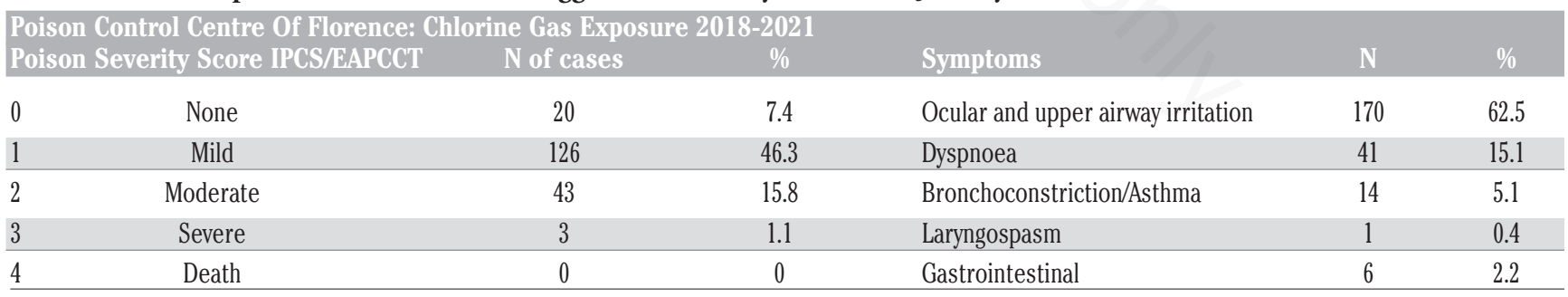

Toxicology Unit \& Poison Control Centre, Azienda Ospedaliero Universitaria Careggi, Florence, Italy between 1 1st January 2018 and $31^{\text {st }}$ October 2021.

Table 3. Indications and suggestions for disposition in exposed patients. Source: Toxicology Unit \& Poison Control Centre, Azienda Ospedaliero Universitaria Careggi, Florence, Italy.

\begin{tabular}{lccccc} 
Poison Control Centre Of Florence: Chlorine Gas Bxposure 2018-2021 & & Therapy & N \\
Patient Disposition & N & $\%$ & None & 67 & 24.6 \\
Observation at home & 102 & 37.5 & 19.1 & Symptomatic or supportive & 205 \\
Family Doctor Evaluation & 52 & 7 & & 75.4 \\
\hline Envoy in Emergency Department & 19 & 24.6 & & \\
Short Observation in Emergency Department & 67 & 11.4 & & \\
\hline Hospital Admission & 31 &
\end{tabular}


charge should include symptom resolution.

Patients with persistent symptoms after a period of observation and supportive treatment should be admitted to the hospital. Depending on symptoms severity (e.g., intubation for pulmonary oedema), an ICU bed may be needed. Criteria for hospital discharge should be improvement or resolution of symptoms. ${ }^{9}$

Depending on the route of exposure and symptoms, it may be appropriate to consult a burn specialist, gastroenterologist, ophthalmologist, or intensivist. For large-scale exposures, public health and hazardous materials personnel should be notified. A poison centre, medical toxicologist, or both should be contacted for moderate to severe exposures.

Anyway, the management of poisoned patients exposed to irritant gases, particularly four aspects that are important for emergency physicians both out-of-hospital and in-hospital management: i) safety: rescuers have to verify to enter a safe scenario (alternatively, a level A or B of PPE should be used) and remove the patient from the source; ii) primary care and survey "ABC": the "A" airway management is critical in these patients and the initial presence of upper airways symptoms as dysphonia, aphonia, stridor or laryngeal spasm indicate prompt intubation; the " $\mathrm{B}$ " breathing management include beta 2 agonists as albuterol for bronchospasm, anti-muscarinic agents as atropine or nebulized glycopyrrolate for airway secretions and PEEP support may be indicated in case of ARDS; the " $C$ " cardiovascular monitoring is needed as dysrhythmias or hemodynamic instability may occur; iii) decontamination: dry and wet decontamination by undressing the patient and by water skin/eye irrigation respectively should be evaluated in cases of mucous/skin symptoms (eyes lacrimation, skin irritation); iv) prolonged clinical observation could be indicated in severely symptomatic chlorine gas exposed patients. Chlorine gas may penetrate lower airways and rarely induce delayed onset ARDS up to 24 hours from acute exposure.

For ingestions, lack of significant initial damage in the oropharynx does not mean deeper, significant gastrointestinal injury cannot develop. Physical exertion can exacerbate symptoms during an ongoing respiratory exposure, as the total exposure will increase with increased minute ventilation. ${ }^{10}$

\section{Conclusions}

Acute accidental inhalation of Chlorine gas is a rare cause of ED arrival, however it can cause important pulmonary disfunctions and even death. Since the symptoms are reversible it is important to perform a quick and appropriate intensive treatment to make sure that the patient does not establish RADS and ARDS.

\section{References}

1. Squadrito GL, Postlethwaite EM, Matalon S. Elucidating mechanisms of chlorine toxicity: reaction kinetics, thermodynamics, and physiological implications. Am J Physiol Lung Cell Mol Physiol 2010;299:289-300.

2. Kim J-A, Yoon S-Y, Cho S-Y, et al. Acute health effects of accidental chlorine gas exposure. Ann Occup Environ Med 2014;26:29.

3. D'Alessandro A, Kuschner W, Wong H, et al. Exaggerated responses to chlorine inhalation among persons with nonspecific airway hyperreactivity. Chest 1996;109:331-7.

4. White CW, Martin JG. Chlorine gas inhalation: human clinical evidence of toxicity and experience in animal models. Proc Am Thorac Soc 2010;7:257-263.

5. Carpenter A, Cox AT, Marion D, et al. A case of a chlorine inhalation injury in an Ebola treatment unit. J R Army Med Corps 2016;162:229-31.

6. White CW, Martin JG. Chlorine gas inhalation: human clinical evidence of toxicity and experience in animal models. Proc Am Thorac Soc 2010;7:257-63.

7. Winder C. The toxicology of chlorine. Environ Res 2001;85:105-14.

8. Yigit O, Soyuncu S, Eray O, et al. Inhalational and dermal injury due to explosion of calcium hypochlorite. Cutan Ocul Toxicol 2009;28:37-40.

9. Mrvos R, Dean BS, Krenzelok EP. Home exposures to chlorine/chloramine gas: review of 216 cases. South Med J 1993;86:654-7.

10. Reisz GR, Gammon RS. Toxic pneumonitis from mixing household cleaners. Chest 1986;89:49-52.

11. Bracco D, Dubois MJ, Bouali R. Intoxication by bleach ingestion (letter). Can J Anesth 2005;52:118-9.

12. Sastre J, Madero MF, Fernandez-Nieto M, et al. Airway response to chlorine inhalation (bleach) among cleaning workers with and without bronchial hyperresponsiveness. Am J Ind Med 2011;54:293-9.

13. Tanen DA, Graeme KA, Raschke R. Severe lung injury after exposure to chloramine gas from houshold cleaners (letter). New Eng J Med 1999;341:848-9.

14. Babl FE, Kharsch S, Woolf A. Airway edema following household bleach ingestion. Am J Emerg Med 1998;16:514-6.

15. De Gennaro L, Brunetti ND, Buquicchio F, et al. Takotsubo cardiomyopathy induced by acute inhalation of hypochlorite drain gel exhalations. Int J Cardiol 2015;180:216-21.

16. Mowry JB, Spyker DA, Cantilena LR Jr, et al. 2013 Annual Report of the American Association of Poison Control Centers' National Poison Data System (NPDS): 31st Annual Report. Clin Toxicol (Phila) 2014;52:1032-283.

17. Mowry JB, et al 2014 Annual Report of the American Association of Poison Control Centers' National Poison Data System (NPDS): 32nd Annual Report. Clin Toxicol 2015;53:962-1147.

18. Casey PB, Dexter EM, Michell J, Vale JA. The prospective value of the IPCS/EC/EAPCCT poisoning severity score in cases of poisoning. J Toxicol Clin Toxicol 1998;36:215-7. 